\title{
Radiation Effects in DMAAS:Cr Ferroelectric Crystal
}

\author{
V. KapustianiK ${ }^{a}, Z$. Czapla ${ }^{b}$, Yu. EliyacheVskyY ${ }^{a}$, \\ YU. KORCHAK ${ }^{a}$, B. KULYK ${ }^{a}$ AND V. RUDYK ${ }^{a}$ \\ ${ }^{a}$ Ferroics Physics Laboratory, Electronics Department \\ Lviv I. Franko National University, Tarnavskogo st., 107, 79017 Lviv, Ukraine \\ ${ }^{b}$ Institute of Experimental Physics, University of Wrockaw \\ pl. M. Borna 9, 50-204 Wrocław, Poland \\ (Received September 11, 2003)

\begin{abstract}
This paper presents the results of investigations of the radiation effects in $\mathrm{NH}_{2}\left(\mathrm{CH}_{3}\right)_{2} \mathrm{Al}\left(\mathrm{SO}_{4}\right)_{2} \times 6 \mathrm{H}_{2} \mathrm{O}$ crystals doped with chromium. On the basis of absorption spectroscopy and resonance Raman scattering study it has been shown that comparatively low doses of radiation first of all causes recharging of $\mathrm{Cr}^{3+}$ on $\mathrm{Cr}^{4+}$ ions. Besides, the processes of dehydrogenation and the changes in arrangement of the hydrogen bond network were observed. These changes are followed by distortion of the complexes bonded by such a type of bonds.
\end{abstract}

PACS numbers: 78.40.Ha, 64.70.Kb, 77.84.--s, 78.30.Hv

\section{Introduction}

Crystals of dimethylammonium aluminum sulfate hexahydrate $\mathrm{NH}_{2}\left(\mathrm{CH}_{3}\right)_{2} \mathrm{Al}\left(\mathrm{SO}_{4}\right)_{2} \times 6 \mathrm{H}_{2} \mathrm{O}$ (DMAAS) have been widely studied recently using different experimental methods [1-7].

In particular a detailed structural investigation was undertaken in order to explain the occurrence of macroscopic polarization in this compound $[8,9]$. It has been ascertained that DMAAS in the paraelectric phase is monoclinic, $P 2_{1} / n$ with the lattice constants $a=6.408 \AA, b=10.752 \AA, c=11.134 \AA$ and $a=100.44^{\circ}[8]$. In this structure, aluminum atoms are coordinated octahedrally by six water molecules which form strong hydrogen bonds $\mathrm{O}-\mathrm{H}$...O (length 2.55-2.70 $\AA$ ) with the oxygens of the sulfate anion, and dimethylammonium cations are disordered [9]. At $T_{\mathrm{C} 1}=152 \mathrm{~K}$ the crystal undergoes a phase transition involving a reorientation of dimethylammonium cations, the structure becomes fully 
ordered and macroscopic polarization appears [6, 9]. In the works [3-5] anomalies of the dielectric and optical parameters at $T_{\mathrm{C} 2}=110.5 \mathrm{~K}$ and $T_{1}=75 \mathrm{~K}$ were found. The low temperature anomaly was supposed to be related to the transition into the antiferroelectric phase, whereas the temperature region between $T_{\mathrm{C} 2}$ and $T_{1}$ was proposed to be characterised by a co-existence of ferroelectric and antiferroelectric phases [5]. An additional phase transition of this system at $390 \mathrm{~K}$ was suggested based on the analysis of optical absorption edge in UV [10].

It has been derived from X-ray investigation that in both paraelectric and ferroelectric phases the six $\mathrm{Al}-\mathrm{O}$ distances are not equal and the differences are more pronounced in the ferroelectric phase, where the $\mathrm{Al}-\mathrm{O}$ distances range from 1.864 to $1.898 \AA$ [9]. The explanation of these results is not straightforward, therefore chromium doped DMAAS crystals were prepared and their spectral properties were investigated [11]. The trivalent chromium ions, with their strong coupling to the surrounding ligands appeared as the convenient probes of symmetry and strength of the crystal field. As it follows from the data of previous spectral investigations $[11,12]$ the symmetry and the structure of the complex around $\mathrm{Cr}^{3+}$ in DMAAS:Cr are very similar to those in ruby crystal [13]. In both cases one can observe the distorted octahedral coordination with only difference that the oxygen atoms serving as the ligands in the ruby are replaced by water molecules in the investigated crystal. Under such circumstances the latter crystals should be considered not only as the model ferroics but also as the model materials of quantum electronics. From this point of view study of the ionizing irradiation of these objects would be interesting both for the fundamental solid state physics and for the practical applications.

As it has been shown in [14] the high doses of $\gamma$-ray irradiation $\left(10^{6}-\right.$ $5 \times 10^{7} \mathrm{R}, \mathrm{Co}-60$ isotope) are followed by a considerable diminishing of the dielectric constant as well as the dielectric dispersion of the undoped DMAAS crystals. Unfortunately, the physical and chemical nature of this phenomenon was not explained. Nevertheless, on the basis of available data one can conclude that the observed considerable diminishing of the low frequency dispersion should be connected with lowering the domain mobility under the influence of irradiation. In the same time, the decrease in dielectric constant values in this case is possibly connected with irreversible radiochemical changes of the sample. Unfortunately, the authors of the above-mentioned paper did not investigate the reversibility of the radiation defects, although it is known that such a type of compounds with organic cation is hardly stable at the used high values of irradiation doses. On the other hand, it is known that such materials with hydrogen bonds appear to be very sensitive to much lower doses of irradiation $[15,16]$ manifesting reversible radiation effects. Under such circumstances it would be convenient to study the influence of low doses of irradiation on the properties of DMAAS:Cr crystals.

It is necessary to note that the spectral methods are very informative and effective in resolving the above-mentioned problem. Moreover, as it was shown 
in the previous investigations [11], the DMAAS crystals doped with chromium appeared to be convenient for a study of small changes of the metal coordination since there is a possibility to investigate the resonance Raman scattering. Indeed, around $\lambda=636 \mathrm{~nm}$ the absorption band in the nonpolarized electronic spectrum was observed. It means that for excitation of the resonance spectra one could use the helium-neon laser $(\lambda=633.8 \mathrm{~nm})$. Moreover, in this case it has been found that the bands corresponding to the vibrations involving the $\mathrm{Cr}^{3+}$ ion and its ligands appeared to be the most intensive. Under such circumstances the comparison of the resonance spectra of the doped sample with the Raman spectra for pure DMAAS crystals would give worth information about the structural changes including those connected with the radiation effects.

\section{Experimental}

Single crystals of $\mathrm{NH}_{2}\left(\mathrm{CH}_{3}\right)_{2} \mathrm{Al}\left(\mathrm{SO}_{4}\right)_{2} \times 6 \mathrm{H}_{2} \mathrm{O}: \mathrm{Cr}^{3+}$ (DMAAS:Cr) were grown from a solution containing aluminum sulfate, excess of dimethylammonium sulfate, and chromium sulfate at a constant temperature of $303 \mathrm{~K}$ by a slow evaporation method. The molar ratio of $\mathrm{Al}^{3+}: \mathrm{Cr}^{3+}$ in solution was equal to 1:0.065. The crystalline platelets of $a$-cut of different thicknesses (about $1.5-3 \mathrm{~mm}$ ) were used in measurements of the absorption spectra. To avoid the influence of absorption water the samples were previously annealed at the temperatures higher than $320 \mathrm{~K}$.

The absorption spectra of DMAAS:Cr in a visible region were recorded at $T=293 \mathrm{~K}$ with an SF-26 absorption spectrophotometer.

The Raman scattering spectra were measured using a computerized DFS-52 M spectrometer employing a phonon counting system. As the source of excitation the He-Ne-laser radiation was used. The spectral resolutions of the spectrometer was $1-2 \mathrm{~cm}^{-1}$.

The sample was irradiated by Co-60 isotope with an activity of $1.5 \mathrm{MBk}$.

\section{Results and discussion}

The nonpolarized absorption spectra of the virgin and irradiated samples of DMAAS:Cr obtained at room temperature (a paraelectric phase) are shown in Fig. 1. These spectra include comparatively wide asymmetric bands that testify to their complex structure. The deconvolution of the bands onto the elementary contours of Gaussian shape was performed using a standard computer program package [17] that considerably simplified the interpretation of the spectra. The low intensity of most bands is caused by low chromium content as well as by the selection rules.

From X-ray investigations data [9] it is known that the $\mathrm{Al}^{3+}$ ions are coordinated octahedrally by six water molecules in the paraelectric phase of DMAAS crystal. The octahedral symmetry $O_{h}$ is accepted as the initial site symmetry for the $\mathrm{Cr}^{3+}$ ions substituting the $\mathrm{Al}^{3+}$ ions. Nevertheless, as it appears from our 


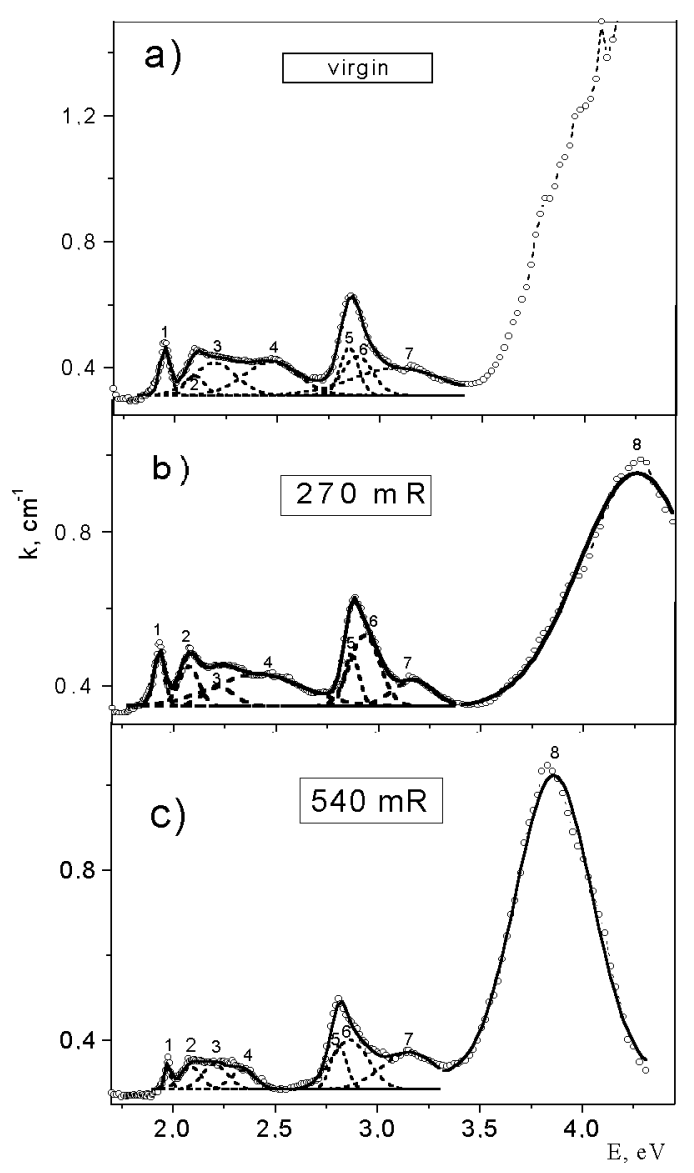

Fig. 1. Optical absorption spectra of DMAAS:Cr crystals obtained at room temperature in nonpolarized light for the virgin sample (a) and the samples irradiated by different doses of radiation (b, c). The solid lines depict the fitting curves resulting from the Gaussians 1-8 shown by dots.

experimental data, the $\mathrm{Cr}\left(\mathrm{H}_{2} \mathrm{O}\right)_{6}^{3+}$ octahedron is at least tetragonally distorted and the site symmetry in this case should be $D_{4 h}$. The corresponding correlation diagram for the energy levels of $\mathrm{Cr}^{3+}$ ions has been presented in [11]. The electron transitions ${ }^{4} A_{2 g}\left({ }^{4} F\right) \rightarrow{ }^{4} T_{2 g}\left({ }^{4} F\right),{ }^{4} A_{2 g}\left({ }^{4} F\right) \rightarrow{ }^{4} T_{1 g}\left({ }^{4} F\right)$ and ${ }^{4} A_{2 g}\left({ }^{4} F\right) \rightarrow{ }^{4} T_{1 g}\left({ }^{4} P\right)$ are parity forbidden but spin allowed. The latest transition should correspond to the comparatively intensive band in the spectra overlapped with a charge transfer band forming the optical absorption edge. In the same time one can note that the number of bands is higher than it is expected from the above-mentioned diagram for the allowed electron transitions. Under such circumstances it is necessary to consider also the spin forbidden transitions between the terms of different multiplicity. In the Tanabe-Sugano diagram for $d^{3}$ ions in the octahedral field at the certain values of the crystal field $D q$ (more exactly $D q / B$, where $B$ is Racah pa- 
rameter) one can observe the crossing points of the ${ }^{4} T_{2 g}\left({ }^{4} F\right),{ }^{4} T_{1 g}\left({ }^{4} F\right)$ terms with the ${ }^{2} E_{g}\left({ }^{2} G\right),{ }^{2} T_{1 g}\left({ }^{2} G\right)$, and ${ }^{2} T_{2 g}\left({ }^{2} G\right)$ terms [18]. The electron transitions from the initial state on the latter doublet levels are spin forbidden. The results of the band identification obtained using both above-mentioned diagrams are presented in Table $\mathrm{I}$.

TABLE I

Identification of the absorption bands in a visible region for DMAAS:Cr crystal.

\begin{tabular}{|c|c|c|c|c|c|}
\hline \multirow[t]{3}{*}{ No } & \multirow{3}{*}{\multicolumn{2}{|c|}{ Transition from ${ }^{4} A_{2 g}\left({ }^{4} F\right)$ on }} & \multicolumn{3}{|c|}{$E, \mathrm{eV}\left(\tilde{\nu}, \mathrm{cm}^{-1}\right)$} \\
\hline & & & \multirow{2}{*}{$\begin{array}{c}\text { Before } \\
\text { irradiation }\end{array}$} & \multicolumn{2}{|c|}{ After irradiation } \\
\hline & & & & $270 \mathrm{mR}$ & $540 \mathrm{mR}$ \\
\hline 1 & \multicolumn{2}{|l|}{${ }^{2} T_{1 g}\left({ }^{2} G\right)$} & $1.95(15725)$ & $1.94(15630)$ & $1.97(15900)$ \\
\hline 2 & \multirow{3}{*}{${ }^{4} T_{2 g}\left({ }^{4} F\right)$} & ${ }^{a} E_{g}$ & $2.10(16890)$ & $2.08(16760)$ & $2.09(16865)$ \\
\hline 3 & & & $2.23(17985)$ & $2.22(17890)$ & $2.20(17730)$ \\
\hline 4 & & $B_{1 \mathrm{~g}}$ & $2.46(19840)$ & $2.42(19500)$ & $2.34(18885)$ \\
\hline 5 & \multirow{3}{*}{${ }^{4} T_{1 g}\left({ }^{4} F\right)$} & ${ }^{b} E_{g}$ & $2.85(22935)$ & $2.86(23045)$ & $2.80(22575)$ \\
\hline 6 & & & $2.89(23310)$ & $2.94(23690)$ & $2.84(22885)$ \\
\hline 7 & & $A_{2 \mathrm{~g}}$ & $3.16(25445)$ & $3.17(25565)$ & $3.15(25380)$ \\
\hline 8 & $\begin{array}{c}{ }^{4} T_{1 g}\left({ }^{4} P\right) \\
\text { Charge transfer or excitonic } \\
\text { transitions of } \mathrm{Cr}^{4+} \text { ion }\end{array}$ & & $>4.10(33000)$ & $4.23(34085)$ & $3.86(31100)$ \\
\hline
\end{tabular}

It has been found that even comparatively low doses of irradiation including both $\gamma$-quanta and $\beta$-particles cause the considerable changes of the spectra in visible and UV regions. In result of irradiation the new band 8 at a high energy part of the spectra is clearly separated from the edge of the intensive band corresponding to the spin allowed transition. Another interesting consequence of the irradiation is suppressing of the almost all bands corresponding to the internal transitions of $\mathrm{Cr}^{3+}$ ion. This tendency is most clearly seen for the higher values of the irradiation doses. It is also important to note that these effects of irradiation practically disappear after keeping of the sample at room temperature during at least 48 hours (see Fig. 2).

Taking into account that the energy diagram of $\mathrm{Cr}^{3+}$ appeared to be very close to those for the ruby crystals one could suggest that irradiation would lead to the similar results. The ionization under the influence of $\gamma$-rays would be followed first of all by recharging of the chromium ions according to the scheme:

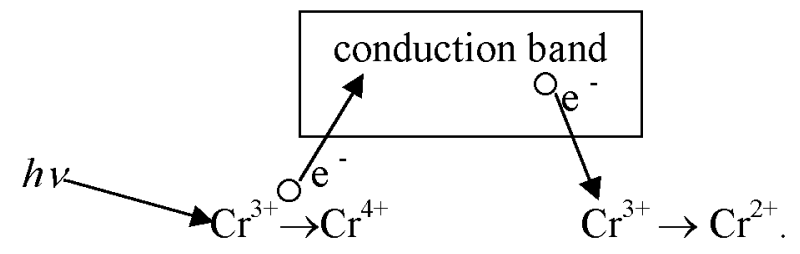




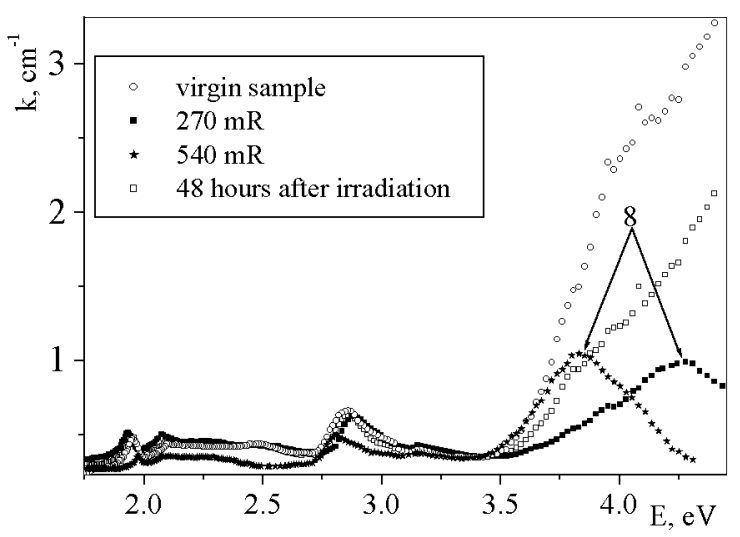

Fig. 2. Comparison of the optical absorption spectra of DMAAS:Cr crystals obtained at room temperature in nonpolarized light for the virgin and irradiated samples and the sample kept during 48 hours after irradiation. The number 8 denotes the new bands arising after irradiation.

The electron is transferred into the conduction band over the ${ }^{2} E$ state of $\mathrm{Cr}^{3+}$ ion. In this case the bands corresponding to the internal transitions should be bleached that is observed in the experiments. Besides, one could expect an additional absorption connected either with $\mathrm{Cr}^{2+}$ or with $\mathrm{Cr}^{4+}$ ions charge transfer transitions. Another possibility would be connected with the formation of the localized exciton state including $\mathrm{Cr}^{4+}$ ion and electron trapped by the ammonium group. Besides, one can consider another type of the exciton states arising when the electrons from the valent orbitals of the oxygen atoms of water molecule are localized on the $\mathrm{Cr}^{3+}$ recharging it to $\mathrm{Cr}^{2+}$. In this case one could also expect the appearance of $\mathrm{O}^{-}$ ions. Nevertheless, such a possibility was excluded at least for the ruby crystals on the basis of the detailed investigations [19]. Moreover, according to [19] the mentioned new band in the region of high energies 4.23 and $3.86 \mathrm{eV}$ (respectively, $\lambda=293$ and $323 \mathrm{~nm}$, depending on the dose of irradiation) would be related to the absorption of $\mathrm{Cr}^{4+}$. The additional absorption connected with $\mathrm{Cr}^{2+}$ in such a type of structures is expected at the energies of $5.07,3.18-3.55$, and $2.59 \mathrm{eV}$ (or at the wavelengths of $245,350-390$, and $480 \mathrm{~nm}$, respectively) and would be hardly divided from the bands of $\mathrm{Cr}^{3+}$ ion. In any case there was not observed any growth of absorption at the above-mentioned energies.

On the other hand, one can note the considerable shift of the new band 8 toward the lower energies observed at growth of the irradiation dose. Such behaviour is characteristic exactly of the excitonic mechanism of the radiation effect [20]. In this case it is clearly seen that the new band "borrows" intensity from the band corresponding to the spin allowed electron transition ${ }^{4} A_{2 g}\left({ }^{4} F\right) \rightarrow{ }^{4} T_{1 g}\left({ }^{4} P\right)$ for $\mathrm{Cr}^{3+}$.

In the same time one can suppose that for the used very low doses of irradiation the formation of displacement defects and corresponding $F$-centres is expected with a much lower probability than the above-mentioned processes. 
TABLE II

Identification of the bands in the Raman spectra of DMAAS:Cr crystal. Geometry of the experiment: $Z(Y Y) X+Z(Y Z) X$.

\begin{tabular}{|c|c|c|}
\hline \multicolumn{3}{|c|}{ Frequencies (wave numbers), $\mathrm{cm}^{-1}$} \\
\hline Virgin sample & $\begin{array}{l}\text { Irradiated } \\
\text { sample }\end{array}$ & $\begin{array}{c}\text { Band } \\
\text { identification }\end{array}$ \\
\hline & 126 & \multirow{3}{*}{ Lattice vibrations } \\
\hline 139 & 142 & \\
\hline 181 & 183 & \\
\hline 226,263 & $216,242,279$ & $\tau\left(\mathrm{CH}_{3}\right)$ \\
\hline \multirow[t]{2}{*}{314} & 316 & $\nu_{6}\left(\mathrm{Cr}-\mathrm{H}_{2} \mathrm{O}\right)$ \\
\hline & 348 & $\nu_{6}\left(\mathrm{Cr}-\mathrm{H}_{2} \mathrm{O}\right)$ \\
\hline 362 & 372 & $\delta(\mathrm{C}-\mathrm{N}-\mathrm{C})$ \\
\hline 393 & 395 & $\delta(\mathrm{C}-\mathrm{N}-\mathrm{C})$ \\
\hline 463 & 462 & $\nu_{2}^{8}$ \\
\hline 513 & 513 & $\nu_{5}\left(\mathrm{Cr}-\mathrm{H}_{2} \mathrm{O}\right)$ \\
\hline 542 & 531 & $\nu_{5}\left(\mathrm{Al}-\mathrm{H}_{2} \mathrm{O}\right)$ \\
\hline- & 551 & $\nu_{2}\left(\mathrm{Cr}-\mathrm{H}_{2} \mathrm{O}\right)$ \\
\hline 574 & 574 & $\nu_{2}\left(\mathrm{Cr}-\mathrm{H}_{2} \mathrm{O}\right)$ \\
\hline 617 & 613 & $\nu_{2}\left(\mathrm{Al}-\mathrm{H}_{2} \mathrm{O}\right)$ or $\nu_{4}^{5}$ \\
\hline 654 & 636,654 & $w\left(\mathrm{H}_{2} \mathrm{O}\right)$ \\
\hline $676,696,737,751,773,796,810$ & $681,712,756,787,807$ & $\rho\left(\mathrm{H}_{2} \mathrm{O}\right)$ \\
\hline 833 & 832 & $\nu_{1}\left(\mathrm{Cr}-\mathrm{H}_{2} \mathrm{O}\right)$ \\
\hline 871 & 870 & $\nu_{1}\left(\mathrm{Al}-\mathrm{H}_{2} \mathrm{O}\right)$ \\
\hline $892,915,938$ & $895,923,943$ & $\nu(\mathrm{C}-\mathrm{N})$ \\
\hline 986 & 988 & $\nu_{1}^{5}$ \\
\hline 1015 & 1032 & $\nu_{3}\left(\mathrm{Me}-\mathrm{H}_{2} \mathrm{O}\right)$ \\
\hline- & 1075 & \\
\hline 1090 & 1110 & $\nu_{3}^{5}$ \\
\hline 1128 & 1131 & $\rho\left(\mathrm{CH}_{3}\right)+\tau\left(\mathrm{NH}_{2}\right)$ \\
\hline
\end{tabular}

s - denotes the vibrations of sulfate groups.

Additional information concerning radiation effects and corresponding structural changes would be drawn from the Raman spectroscopy which depending on the geometry of experiment possesses resonance or so-called pre-resonance character. In Fig. 3 the Raman spectra for the virgin and irradiated samples are compared. These spectra for the chosen geometry possess the pre-resonance character. Their identification for the virgin sample was performed in [11]. Although the irradiation do not change drastically the above-mentioned spectra (Table II) one can note the following changes: 
1. clear splitting of the bands corresponding to the torsional vibration of $\mathrm{CH}_{3}$ group;

2. the band corresponding to $\nu_{6}\left(\mathrm{Cr}-\mathrm{H}_{2} \mathrm{O}\right)$ mode is shifted toward higher frequencies;

3 . the $\nu_{2}^{\mathrm{s}}$ mode of sulfate complex is broadened and shifted toward higher frequencies;

4. the vibration of water molecules ( $w$ - and $\rho$-type) are shifted toward higher frequencies;

5 . the $\nu_{1}^{\mathrm{s}}$ mode of sulfate complex is broadened;

6. the valence vibrations $\nu_{3}\left(\mathrm{Cr}-\mathrm{H}_{2} \mathrm{O}\right)$ are shifted toward higher frequencies;

7. more pronounced splitting of $\nu_{3}^{\mathrm{s}}$ mode of sulfate complex;

8. practically all modes corresponding to the skeletal $\mathrm{C}-\mathrm{N}-\mathrm{C}$ vibrations are slightly shifted toward higher frequencies.

The observed effects practically are reversible. This conclusion is confirmed by Fig. 3 b which as an example presents the low frequency part of the Raman spectra for virgin and irradiated samples as well as the spectra obtained after 48 hours after irradiation.
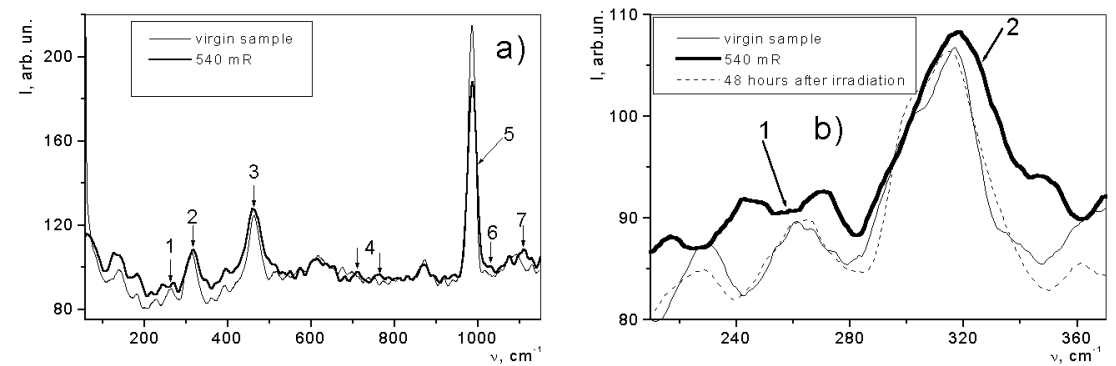

Fig. 3. Raman spectra of the virgin and irradiated samples of DMAAS:Cr obtained at room temperature (a) and the low frequency part of the same spectra including also those for the sample kept during 48 hours after irradiation (b). Orientations and the corresponding modes: $Z(Y Y) X+Z(Y Z) X$.

The above-mentioned experimental facts (the items 2, 4, and 6) fairly well correlate with the model based on the recharging of the chromium ion. One could expect that the bonds of $\mathrm{Cr}^{4+}$ ion with ligands would be stronger than for $\mathrm{Cr}^{3+}$. In such a case the frequency of the metal-hydrate complexes internal vibrations (first of all valence vibrations) are expected to be higher.

On the other hand, under the influence of comparatively low doses of irradiation first of all one could observe the dehydrogenation process which would influences the vibrations of methyl groups and water molecule (items 1,4). As it 
is clearly seen from Fig. 3b the effect is reversible. It is not odd taking into account a high mobility of protons especially in such compounds with comparatively high protonic conductivity [11]. Unfortunately it was impossible to observe the dehydrogenation effect for other types of organic groups vibrations due to comparatively intensive phosphorescence observed at higher frequencies. One can also suppose that the observed frequency shift of both deformation and valence skeletal vibrations of the dimethylammonium group (item 8) would be related to the breaking of the hydrogen bonds (including $\mathrm{N}-\mathrm{H}$...O bonds) with their following relaxation to a certain new structure. It would change the degree of disordering of the dimethylammonium complexes that are only partially ordered at room temperature.

Another interesting effect of irradiation concerns the sulfate ion. According to the items $3,5,7$ this complex is considerably distorted obviously through its hydrogen bonds with the metal-hydrate complexes which are strongly affected by irradiation.

\section{Conclusion}

As a result of performed investigations it has been shown that $\mathrm{Cr}^{3+}$ ions appeared as a convenient probe for the study of structural changes in the DMAAS crystals arising under the influence of different external factors. On the basis of absorption spectroscopy and the Raman scattering study it was found that comparatively low doses of radiation first of all cause recharging of $\mathrm{Cr}^{3+}$ on $\mathrm{Cr}^{4+}$ obviously with the formation of the localised exciton states. On the other hand, the processes of dehydrogenation occur in result of irradiation. Moreover, the changes in the arrangement of the hydrogen bond network are observed that leads to the distortion of the complexes bonded by such a type of bonds. All observed effects are found to be practically reversible although for the full returning of the sample toward the pre-radiation state at least thirty days are necessary.

\section{Acknowledgment}

This work was supported by Józef Mianowski Fund (Republic of Poland).

\section{References}

[1] L. Kirpichnikova, E. Andreyev, N. Ivanov, L. Shuvalov, V. Varikash, Kristallografiya 33, 1437 (1988).

[2] O. Vlokh, V. Kapustianik, I. Polovinko, S. Sveleba, V. Varikash, E. Andreyev, $Z h$. Prikl. Spektrosk. 51, 854 (1989).

[3] O.G. Vlokh, V.B. Kapustianik, I.I. Polovinko, E.F. Andreyev, V.M. Varikash, L.A. Shuvalov, Ferroelectrics 111, 333 (1990).

[4] V. Kapustianik, M. Bublyk, I. Polovinko, S. Sveleba, Z. Trybula, Phase Transit. 49, 231 (1994). 
[5] V. Kapustianik, S. Sveleba, I. Stasyuk, O.Velychko, Z. Czapla, R. Tchukvinskyi, Phys. Status Solidi B 228, 785 (2001).

[6] V. Kapustianik, M. Fally, H. Kabelka, H. Warhanek, J. Phys., Condens. Matter 9, 723 (1997).

[7] A. Pietraszko, K. Łukaszewicz, L.F. Kirpichnikova, Polish J. Chem. 67, 1877 (1993).

[8] N. Galešic, V.B. Jordanovska, Acta Crystallogr. C 48, 256 (1992).

[9] A. Pietraszko, K. Łukaszewicz, Polish J. Chem. 68, 1239 (1994).

[10] O.G. Vlokh, V.B. Kapustianyk, I.I. Polovynko, I.A. Mykhalyna, S.A. Sveleba, E.F. Andreyev, V.M. Varikash, L.A. Shuvalov, Kristallografiya 36, 919 (1991).

[11] V. Kapustianik, Z. Czapla, R. Tchukvinskyi, A. Batiuk, Yu. Eliyashevskyi, Yu. Korchak, V. Rudyk, Phys. Status Solidi A, on line publication.

[12] W. Ryba-Romanowski, S. Gołąb, G. Domoniak-Dzik, W.A. Pisarski, D. Podsiadła, Z. Czapla, J. Mol. Struct. 450, 219 (1998).

[13] S.V. Grum-Grzhymailo, L.B. Pasternak, D.T. Sviridov, L.G. Chentsova, M.A. Chernyshova, in: The Crystal Spectroscopy, Nauka, Moscow 1966, p. 168 (in Russian).

[14] A.U. Sheleg, K.V. Iodkovskaya, N.F. Kurilovich, Proc. Byelarus Acad. Sci. Ser. Phys. Math. Sci. No 2, 111 (2001).

[15] V. Kapustianik, S. Sveleba, Ya. Ozhybko, R. Tchukvinskyi, V. Mokryi, V. Soldatov, I. Polovynko, J. IEEE 4, 801 (1994).

[16] V. Kapustianik, Ukr. Phys. J. 43, 343 (1998).

[17] V.B. Kapustianik, V.V. Bazhan, Yu.M. Korchak, Phys. Status Solidi B 234, 674 (2002).

[18] A.B.P. Lever, Inorganic Electronic Spectroscopy, Elsevier Science Publ. B.V., Amsterdam 1984.

[19] G.E. Archangelskii, Z.L. Morgenshtern, Phys. Status Solidi 22, 289 (1967).

[20] M.A. Elango, Elementary Nonelastic Radiation Processes, Nauka, Moscow 1988 (in Russian). 